\title{
Finite element analysis of the femur during stance phase of gait based on musculoskeletal model simulation
}

\author{
Jeong-Woo Seo ${ }^{\mathrm{a}}$, Dong-Won Kang ${ }^{\mathrm{a}}$, Ju-Young Kim ${ }^{\mathrm{a}}$, Seung-Tae Yang ${ }^{\mathrm{a}}$, Dae-Hyeok Kim ${ }^{\mathrm{a}}$, \\ Jin-Seung Choi ${ }^{\mathrm{a}, \mathrm{b}}$ and Gye-Rae Tack ${ }^{\mathrm{a}, \mathrm{b},{ }^{*}}$ \\ ${ }^{a}$ Department of Biomedical Engineering, College of Biomedical \& Health Science, Konkuk University, \\ 322 Danwoldong, Chungju, Korea \\ ${ }^{b}$ BK21 Plus Research Institute of Biomedical Engineering, Konkuk University, 322 Danwoldong, \\ Chungju, Korea
}

\begin{abstract}
In this study, the accuracy of the inputs required for finite element analysis, which is mainly used for the biomechanical analysis of bones, was improved. To ensure a muscle force and joint contact force similar to the actual values, a musculoskeletal model that was based on the actual gait experiment was used. Gait data were obtained from a healthy male adult aged 29 who had no history of musculoskeletal disease and walked normally ( $171 \mathrm{~cm}$ height and $72 \mathrm{~kg}$ weight), and were used as inputs for the musculoskeletal model simulation to determine the muscle force and joint contact force. Among the phases of gait, which is the most common activity in daily life, the stance phase is the most affected by the load. The results data were extracted from five events in the stance phase: heel contact (ST1), loading response (ST2), early mid-stance (ST2), late mid-stance (ST4), and terminal stance (ST5). The results were used as the inputs for the finite element model that was formed using $1.5 \mathrm{~mm}$ intervals computed tomography (CT) images and the maximum Von-Mises stress and the maximum Von-Mises strain of the right femur were examined. The maximum stress and strain were lowest at the ST4. The maximum values for the femur occurred in the medial part and then in the lateral part after the mid-stance. In this study, the results of the musculoskeletal model simulation using the inverse-dynamic analysis were utilized to improve the accuracy of the inputs, which affected the finite element analysis results, and the possibility of the bone-specific analysis according to the lapse of time was examined.
\end{abstract}

Keywords: Musculoskeletal modeling, finite element analysis, inverse dynamics

\section{Introduction}

Hip arthroplasty or internal fixation of hip fracture recently started to be performed to recover the function of the hip joint or femur fractured by aging, disease, or injury [1]. In the procedure, the most important factor is the strength limit of used implant and the bone. Because the actual load applied inside the body cannot be measured, finite element analysis (FEA) is used as an indirect method. It can cover the internal stress of a complex-shaped object and the stress on the surface [2], and has high

\footnotetext{
${ }^{*}$ Corresponding author: Gye-Rae Tack, Department of Biomedical Engineering, College of Biomedical \& Health Science, Konkuk University, 322 Danwoldong, Chungju, Korea. Tel.: 82-840-3762; Fax: 82-43-851-0620; E-mail: grtack@kku.ac.kr.
}

0959-2989/14/\$27.50 @ 2014 - IOS Press and the authors. 
geometrical accuracy. In addition, it has good results even for complex material characteristics. Therefore, it is used for bone assessment. Various preceding studies that used FEA have been conducted to examine the mechanical characteristics of the femur. Jiang [3] studied how to find an artificial femur head model closest to the actual femur in terms of its mechanical characteristics. In addition, he compared the force of the hip joint according to the gait phase as the load condition for the FEA, and verified that the peak joint force was highest in the heel strike phase. Andreaus [2] created an FEA model with the same condition as that of the in-vivo model of Kassi [4] to assess the functions of a patient who had undergone artificial hip arthroplasty in various daily activities. Sverdlova [5] conducted FEA of the femur using the hip joint contact force and the muscle force from the study results of Bergmann [6] and Brand [7] as input values to verify that the compressive stress was concentrated on the diaphyseal and proximal areas of the femur. As in the preceding studies, the functions and characteristics of a long bone, such as the femur, must first be considered to ensure accurate assessment of the load and boundary conditions. It is known that a long bone produces a large tensile load because of the large bending moment. Thus, bone is influenced by the bending moment, and the muscle plays an important role in reducing the effect [7]. The muscle absorbs the bending moment and mitigates the load on the bone. In the case of indirect measurement, the type and force of the muscle activated by the human motion must be accurately considered. The representative study of this is done by Duda [8], which examined the difference in the mechanical characteristics according to the method to consider the type of muscle that worked for the femur. The results of the study showed that the tensile strain and the compressive strain were overestimated if all the muscle forces were not considered.

When the indirect measurement method is used, it is important to reproduce the various conditions such as the muscle force, joint contact force, and physical characteristics of the bone, and they must be used to estimate the results under more detailed exercise conditions. In this study, the results of the inverse dynamic musculoskeletal model simulation were used to improve the accuracy of the inputs that affected the FEA results. The stance phase is the most affected by the load among the phases of the gait which is the most common activity in daily life. Thus, this was divided into five stages to verify the possibility of the bone characteristic analysis according to the lapse of time in different dynamic activities.

\section{Method}

\subsection{Subject}

The study was conducted on healthy male adult aged 29 who had no history of musculoskeletal disease and walked normally $(171 \mathrm{~cm}$ height and $72 \mathrm{~kg}$ weight).

\subsection{Experiment}

A 3D motion analysis system with six infrared cameras (Motion Analysis Corp., USA) and two force plates (AMTI, USA) were used to acquire the motion and ground reaction force data at $100 \mathrm{~Hz}$ and $1,000 \mathrm{~Hz}$ [9]. Twenty reflective markers were attached as plug-in sets, and the gait was repeatedly conducted three times with preferred walking speed (Figure 1). 

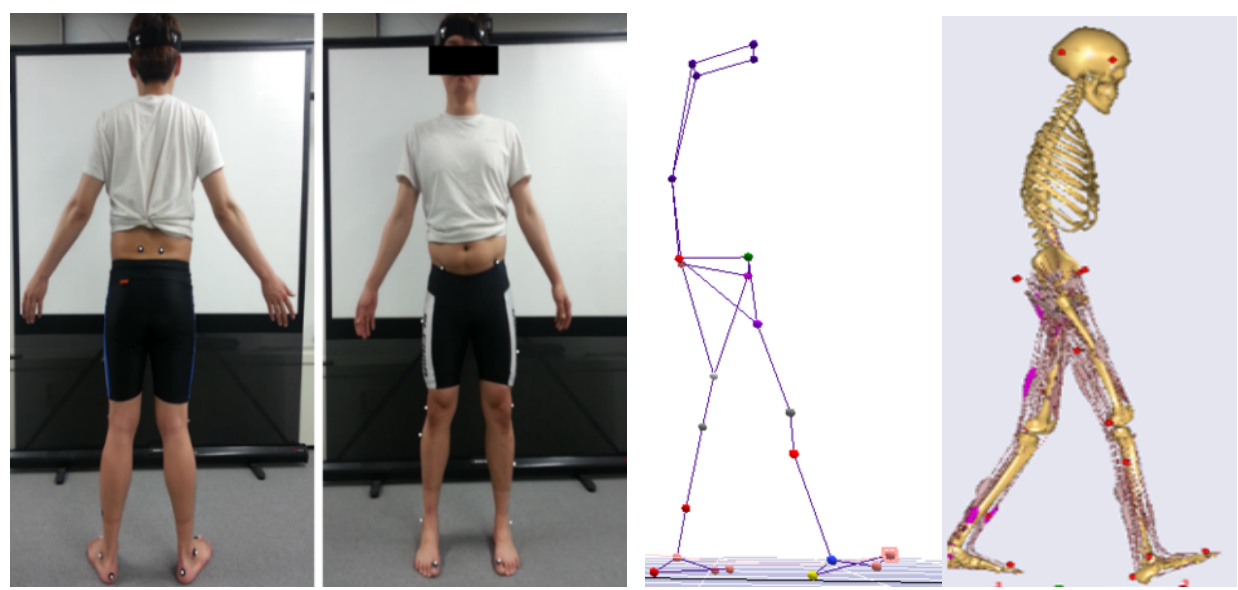

Fig. 1. Plug-in marker set $\&$ motion data with Anybody MS model.

\subsection{Musculoskeletal model simulation}

To estimate the load on the femur during the actual gait by determining the muscle force used as the input value for the FEA, the musculoskeletal simulation program Anybody Modeling System (Anbody Technology, Inc., Denmark) was used. The force and direction of the activated muscle were determined based on the actual gait data. The human body model was the hill-type muscle-based gait lower-extremity model, which had 56 muscles split into 176. In this study, 18 muscles were selected, which were joined to the femur and showed significant activation during the gait activity.

\subsection{Gait event selection}

From the stance phase of the gait, the data for the following five points were extracted: heel contact (ST1), loading response (ST2), early mid-stance (ST3), late mid-stance (ST4), and terminal stance (ST5). ST1 was set when the height of the heel marker at the heel of the foot was at the lowest point of the right heel contact in the position data from the 3D motion analyzer, with a start and end points of a stride set as $0 \%$ and $100 \%$, respectively. ST2 was set when the foot became flat with an anterior ground reaction force vector, moving up toward the hip (10\% of the gait cycle). ST3 was set when the vertical axis was starting to be at the lowest; that is, when it passed the mid-foot, the foot became flat with the ankle neutral, and the ground reaction vector was minimally displaced from the joint center ( $15 \%$ of the gait cycle). ST4 was set for the duration just before the vertical axis height of the heel marker rose. At this point, the ankle was dorsiflexed, and the vector was anterior to the knee and ankle (30\% of the gait cycle). Finally, ST5 was set for the free forward fall of the body $(50 \%$ of the gait cycle). The ground reaction force data were extracted from the five stages [10].

\subsection{Finite element analysis}

To create the FEA model for the subject who participated in the gait experiment, Mimics v13 (Materialise Inc., Belgium), an anatomy software program for medical image segmentation for engineering, was used. The right femur was formed based on CT images with $1.5 \mathrm{~mm}$ intervals. ANSYS v11 (ANSYS Inc., USA), FEA software program, was used to create and analyze the grids of the femur model. The grids were $2 \mathrm{~mm}$ tetrahedral elements that consisted of 30,163 nodes and 17,220 elements. The 
femur was assumed to be homogeneous and isotropic, and linearly and elastically deformed. It was divided into the cortical bone, which supported most of the femur load, and the cancellous bone, which was less dense, and the typical Young's modulus was used to them $(17,000 \mathrm{MPa}$ and 1,500 $\mathrm{MPa}$, respectively) [11,12]. The same Poisson's ratio (0.33) was used [12]. The variables were the maximum Von-Mises stress, maximum Von-Mises strain, and total deformation, which represented the maximum distortion energy. The individual muscle forces were reproduced in the similar form of the anatomical attachment and refer to the CT-image. The 3-dimensional contact forces were applied to a vector value as shown in Figure 2.

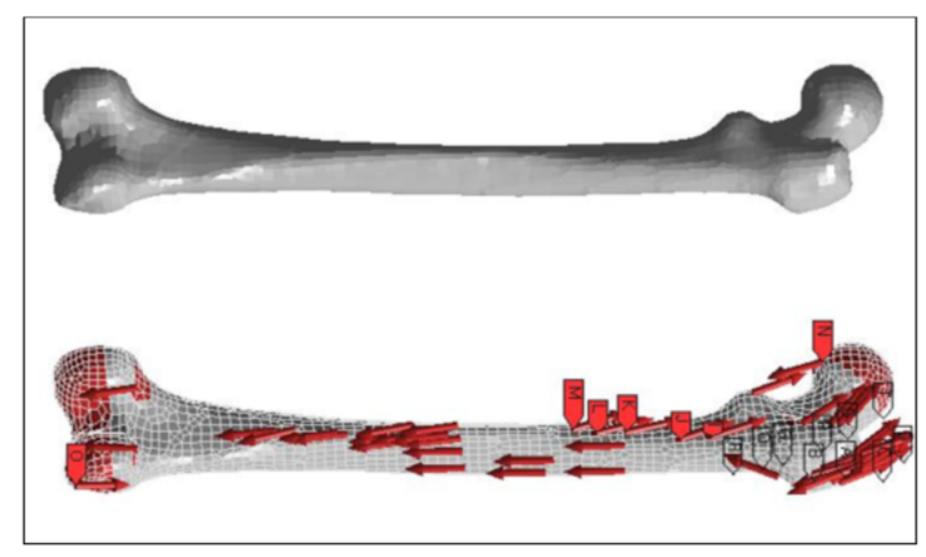

Fig. 2. Individual muscle \& contact forces.
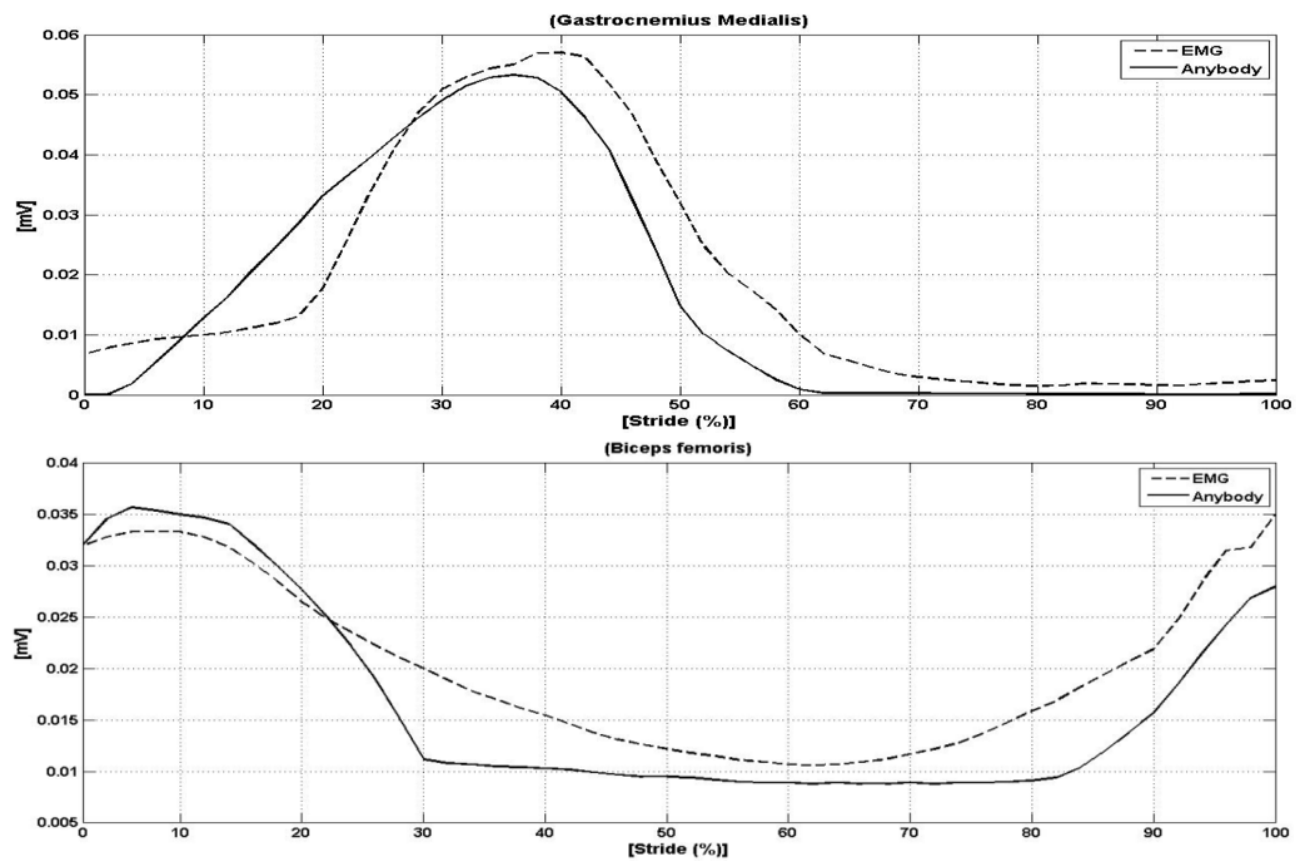

Fig. 3. Anybody vs. EMG results. 


\subsection{Musculoskeletal model simulation result}

To verify the Anybody modeling system output, the EMG (Gastrocnemius medialis \& Biceps femoris) was measured during normal walking. Similar results were shown when compared with Anybody Outputs (Figure 3).

Table 1 shows the muscle force and joint contact force according to the five stance phase events from the musculoskeletal model simulation using the actual gait experiment.

\subsection{Finite element analysis result}

The FEA was conducted by applying the muscle force and joint contact force calculated from the inverse dynamic musculoskeletal model simulation to the CT-image based right femur model. Figure 4 shows the max Von-Mises stress and max Von-Mises strain at five stance phase stages. The area most influenced by the load moved from the medial part of the femur to the lateral part, starting from the ST3. Figure 5 shows the resulting max Von-Mises stress, max Von-Mises strain, and total deformation in Figure 4. The max Von-Mises stress, max Von-Mises strain, and total deformation were 151.4 MPa, $7.571 \times 10^{-4} \mathrm{~mm} / \mathrm{mm}$, and $3.178 \mathrm{~mm}$, respectively at ST1; $141.3 \mathrm{MPa}, 7.062 \times 10^{-4} \mathrm{~mm} / \mathrm{mm}$, and $2.981 \mathrm{~mm}$ at ST2; $114.4 \mathrm{MPa}, 5.718 \times 10^{-4} \mathrm{~mm} / \mathrm{mm}$, and $2.383 \mathrm{~mm}$ at ST3; $111.4 \mathrm{MPa}, 5.570 \times 10^{-4}$ $\mathrm{mm} / \mathrm{mm}$, and $2.335 \mathrm{~mm}$ at ST4; and $114.4 \mathrm{MPa}, 5.718 \times 10^{-4} \mathrm{~mm}$, and $2.390 \mathrm{~mm}$ at ST5. Thus, the max Von-Mises stress, max Von-Mises strain, and total deformation were highest at ST1 and lowest at ST4.

Table 1

Muscle and hip joint contact forces at the subdivided stance phase of a gait event (unit: N)

\begin{tabular}{llllll}
\hline Gait Phase Muscle & $\begin{array}{l}\text { Heel Con- } \\
\text { tact (ST1) }\end{array}$ & $\begin{array}{l}\text { Loading } \\
\text { Response (ST2) }\end{array}$ & $\begin{array}{l}\text { Early Mid- } \\
\text { stance (ST3) }\end{array}$ & $\begin{array}{l}\text { Late Mid- } \\
\text { stance (ST4) }\end{array}$ & $\begin{array}{l}\text { Terminal } \\
\text { Stance (ST5) }\end{array}$ \\
\hline \hline Add magnus & 180 & 102 & 0 & 0 & 0 \\
\hline Gluteus maxi & 452 & 432 & 406 & 395 & 389 \\
\hline Gluteus medi & 848 & 862 & 840 & 851 & 853 \\
\hline Gluteus mini & 542 & 563 & 516 & 540 & 582 \\
\hline Iliacus & 0 & 0 & 0 & 0 & 84 \\
\hline Inferior gemeli & 58 & 60 & 62 & 50 & 32 \\
\hline Superior gemeli & 53 & 54 & 54 & 55 & 60 \\
\hline Obturatorexter & 22 & 23 & 27 & 29 & 32 \\
\hline Obturator inter & 422 & 410 & 404 & 413 & 435 \\
\hline Piriformis & 198 & 192 & 180 & 185 & 193 \\
\hline Quadratusfemoris & 25 & 23 & 17 & 22 & 28 \\
\hline Biceps femoris & 185 & 155 & 115 & 138 & 165 \\
\hline Ten faslatae & 24 & 18 & 14 & 11 & 9 \\
\hline Gastro-lateral & 370 & 370 & 372 & 385 & 390 \\
\hline Gastro-medial & 540 & 534 & 530 & 546 & 562 \\
\hline Vastuslateralis & 13 & 12 & 11 & 9 & 7 \\
\hline Vastusmedialis & 4 & 4 & 2 & 2 & 2 \\
\hline Vastusintermedius & 8 & 6 & 2 & 4 & 5 \\
\hline \hline Total muscle force & 3,944 & 3,820 & 3,552 & 3,635 & 3,828 \\
\hline Hip joint contact force & 627.16 & 699.79 & 1162 & $1,390.1$ & $1,418.6$ \\
\hline Patello femoral joint contact force & 752.52 & 787.96 & $1,177.2$ & $1,455.7$ & $1,491.2$ \\
\hline
\end{tabular}




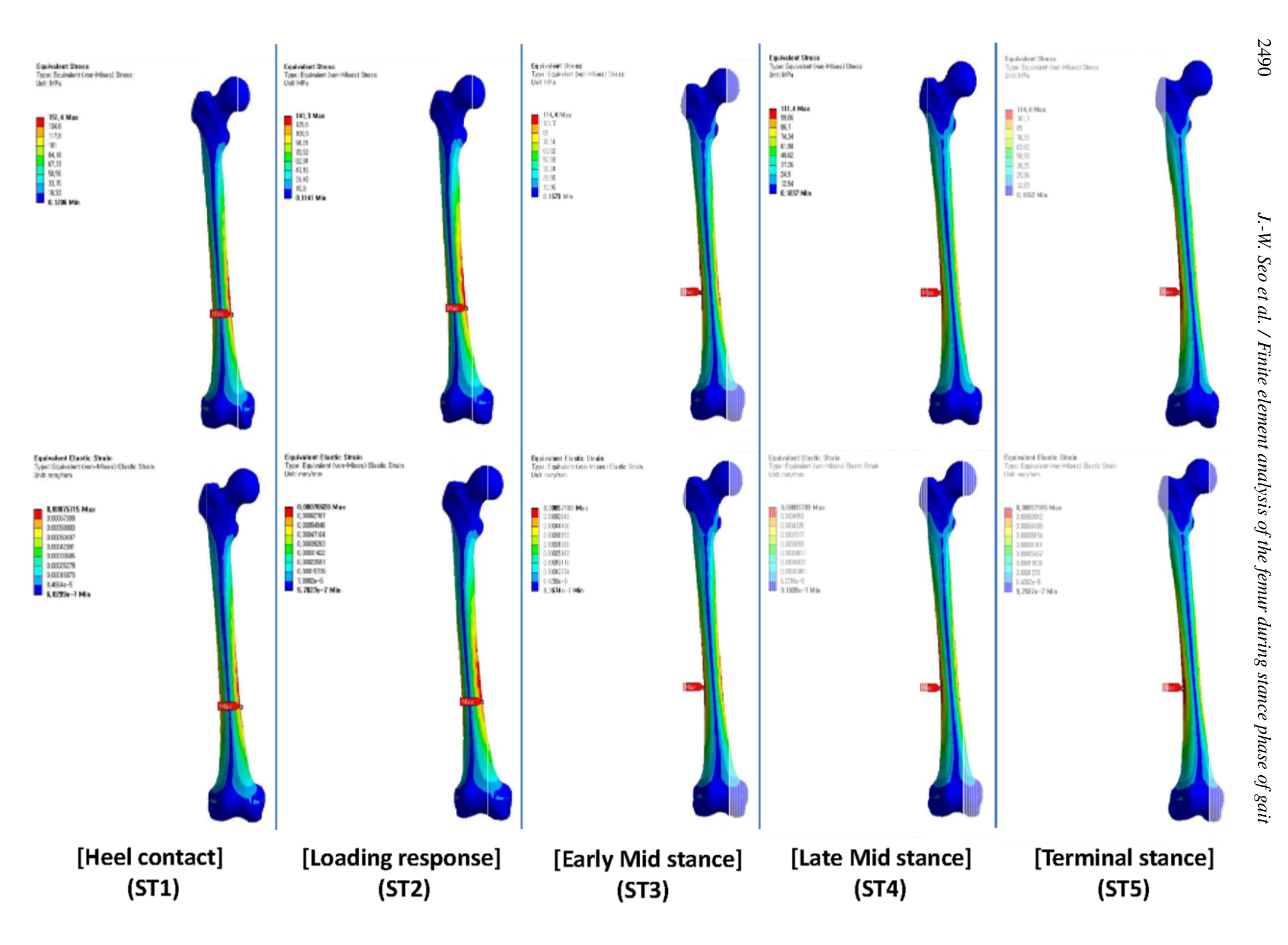



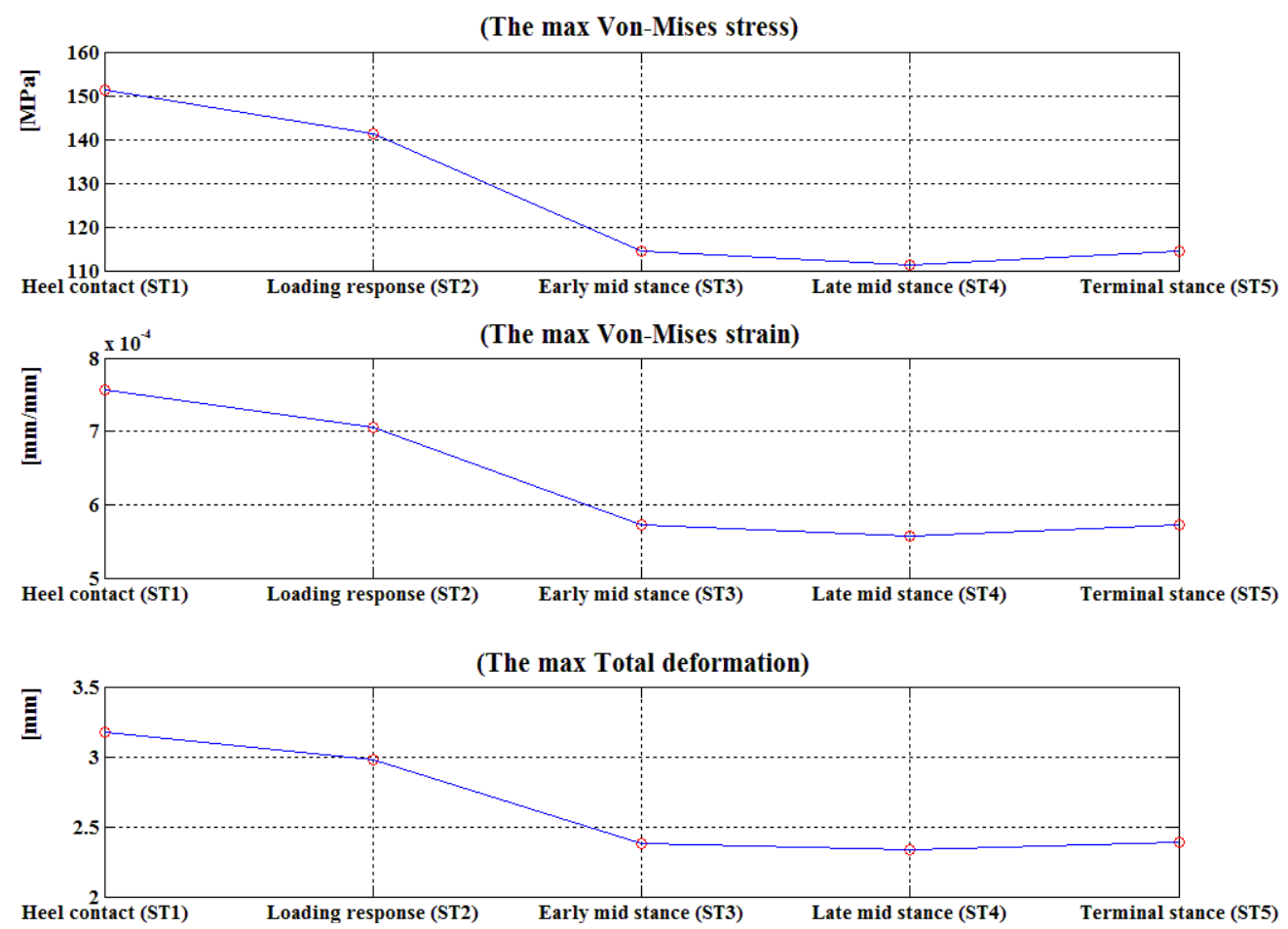

Fig. 5. Results of FEA (Max Von-Mises stress, strain \& Total deformation.

\section{Discussion and conclusion}

In this study, the accuracy of the inputs for the FEA, which are used for the biodynamic analysis of bones, were improved. To ensure that the muscle force and joint contact force are similar to the actual values, the musculoskeletal model simulation that was based on the actual gait experiment was used.

The stance phase, which is the most influenced by the load among the phases of the gait (which is the most common activity in daily life), was divided into five stages according to the lapse of time to verify the effect of the load on the femur. For the examination of the external force on the femur in the FEA results, the max Von-Mises stress and Von-Mises strain were determined. The Von-Mises stress represents the max distortion energy produced by stress factors at the appropriate point of an object and is used to estimate the fracture of objects. The force of individual muscle is necessary for the examination of the load applied to the bone. That is, the role of the muscle varies according to the phase. For example, in the actual gait phase, the hamstring muscles and quadriceps muscles are mainly used for hip flexion and knee extension at the heel contact phase. At the mid-stance, the use of the gluteus muscle and the gastrocnemius muscle increases for the hip joint adduction and the prevention of dorsiflexion, respectively, and the activity of the other muscles somewhat decreases. In addition, the activity of most of the leg muscles other than the gluteus medius muscle relatively decreases. At the terminal stance, the activity of the calf muscles decreases as the hip joint and knee are bent, and the rectus femoris muscle and adductor longus muscle are activated [10,13]. In most preceding studies, the muscle force, which is the input for the FEA, is simplified or omitted. However, it can cause overesti- 
mation of the FEA results [8]. Therefore, this study is advantageous because it used the musculoskeletal model to consider diverse muscle forces and joining points on the femur.

The stance phase is where the center of the body weight crosses the center of the sole on the ground, and one leg supports the body weight and supports more weight than in the swing phase. Thus, in view of the center of pressure (COP), the stress on the femur at the stance phase is also greater $[8,12]$. In this study, the stance phase was divided into five stages according to the COP. In the mid-stance phase, the Von-Mises stress and strain were lower than in the other phases, because the muscles that had been activated in the heel contact phase had a less significant effect and the stress and strain on the femur decreased. After the mid-stance, the muscle activation that had been lowered before the terminal stance was restarted, and the stress and strain on the femur became greater again. In addition, it was anticipated that a smaller total of all muscle forces would reduce the maximum stress and strain. However, the results of the study showed that the Von-Mises stress and strain and the total deformation were lowest at ST4, although the total muscle force was lowest at ST3, which was due to the effect of the hip and patello-fermoral contact force that increased with the lapse of time in the stance phase [13], which be investigated in further study.

The femur area in this study that was influenced by the max Von-Mises stress and max Von-Mises strain was the same as the ventral and lateral parts of the femur. The result was the same as the estimated stress and strain distribution of the mid-stance phase in the preceding studies of Duda [8] and Taylor [14], which implies that the femur was influenced by the bending moment and resulted from the morphological characteristics of the bent femur shaft and the considerably biased femur head [14]. In addition, the max area was the medial area in the loading response, but it was the lateral area in the mid-stance, which was due to that the center of the body weight was in the frontal-lateral direction in the stance phase [10].

To obtain results as accurate as actual values under the assumption that the invasive approach cannot be used for the FEA of bones, the optimized load that is the same as the femur condition of the subject must be reproduced. In addition, the effect on the bone of diverse motions such as running, climbing the stairs, and sitting to standing can be examined as in the stance phase, which was divided into several stages. Based on the results, it is expected that the load limit of the artificial joint can be estimated or that basic data for an ergonomic design can be provided.

\section{Acknowledgement}

This work was supported by Konkuk University.

\section{References}

[1] C. Du, H. Ma, M. Rou, Z. Zhang, X. Yu and Y. Zeng, An experimental study on the biomechanical properties of the cancellous bones of distal femur, Bio-Medical Materials and Engineering 16 (2006), 215-222.

[2] U. Andreaus, M. Colloca and A. Toscano, Mechanical behavior of a prosthesized human femur: a comparative analysis between walking and stair climbing by using the finite element method, Biophysics and Bioengineering Letters 10 (2008), 1-15.

[3] H.B. Jiang, Static and dynamic mechanics analysis on artificial hip joints with different interface designs by the finite element method, Journal of Bionic Engineering 4 (2007), 123-131.

[4] J.P. Kassi, M.O. Heller, U. Stoeckle, C. Perka and G.N. Duda, Stair climbing is more critical than walking in preclinical assessment of primary stability in cementless THA invitro, Journal of Biomechanics 38 (2005), 1143-1154. 
[5] N.S. Sverdlova and U. Witzel, Principles of determination and verification of muscle forces in the human musculoskeletal system: Muscle forces to minimize bending stress, Journal of Biomechanics 43 (2010), 387-396.

[6] G. Bergmann, G. Deuretzabacher, M. Heller, F. Graichen, A. Rohlmann and J. Strauss, Hip forces and gait patterns from rountine activities, Journal of Biomechanics 34 (2001), 859-871.

[7] R.A. Brand, D.R. Pedersen and J.A. Friederich, The sensitivity of muscle force predictions to changes in physiologic cross-sectional area, Journal of Biomechanics 19 (1986), 589-596.

[8] G.N. Duda, E. Schneider and E.Y.S. Chao, Internal forces and movements in the femur during walking, Journal of Biomechanics 30 (1997), 933-941.

[9] T. Hattori, Body up-down acceleration in kinematic gait analysis in comparison with the vertical ground reaction force, Bio-Medical Materials and Engineering 8 (1998), 145-154.

[10] J.P. Perry, Gait Analysis: Normal and Pathological Function, SLACK Inc., New Jersey, 1992, pp. 149-167.

[11] D.T. Reilly, A.H. Berrstein and V.H. Frankel, The elastic modulus for bone, Journal of Biomechanics 7 (1974), 271275.

[12] T.M. Keaveny and W.C. Hayes, A 20-year perspective on the mechanical properties of trabecular bone, Journal of Biomechanical Engineering 115 (1993), 534-542.

[13] D.R. Pedersen, R.A. Brand and D.T. Davy, Pelvic muscle and acetabular contact forces during gait, Journal of Biomechanics 30 (1997), 959-965.

[14] M.E. Taylor, K. E. Tanner, M.A.R. Freeman and A.L. Yettram, Stress and strain distribution within the intact femur: Compression or bending?, Medical Engineering \& Physics 18 (1996), 122-131. 